\title{
Management of Chronic Expanding Haematoma Using Triamcinolone after Latissimus Dorsi Flap Harvesting
}

\author{
Mariko Hamada*, Yusuke Shimizu*, Noriko Aramaki-Hattori, Tatsuya Kato, Keiko Takada, \\ Marie Aoki, Kazuo Kishi, Tomohisa Nagasao \\ Department of Plastic and Reconstructive Surgery, Keio University School of Medicine, Tokyo, Japan
}

\begin{abstract}
Chronic expanding haematoma (CEH) is a rare type of haematoma that enlarges slowly and continuously without coagulation. It can occur following surgery because of shear stressinduced bleeding in the scar tissue between the subcutaneous fat and fascia. We present three cases of large chronic CEH that were successfully treated with triamcinolone injections. Three female patients developed large chronic CEH at 9 months, 5 years, and 6 years, respectively, after latissimus dorsi flap harvesting for breast reconstruction. Although the condition did not improve after multiple sessions of haematoma aspiration in the first two patients, it resolved following a single $40-\mathrm{mg}$ triamcinolone injection along with appropriate compression dressing for several weeks. In the third patient, triamcinolone was injected immediately after the initial aspiration of the haematoma, and the condition improved considerably. There were no side effects in any of the patients. To the best of our knowledge, this is the first report of successful treatment of large $\mathrm{CEH}$ using triamcinolone. Therefore, we suggest that triamcinolone injections be considered for the treatment of $\mathrm{CEH}$.
\end{abstract}

Keywords Haematoma / Triamcinolone / Free tissue flaps / Seroma
Correspondence: Yusuke Shimizu Department of Plastic and Reconstructive Surgery, Keio University School of Medicine, 35 Shinanomachi, Shinjuku, Tokyo 160-8582, Japan Tel: $+81-03-5363-3814$ Fax: +81-03-3352-1054 E-mail:yyssprs@gmail.com

${ }^{*}$ Contributed equally to this work and are co-first authors.

No potential conflict of interest relevant to this article was reported.

\section{INTRODUCTION}

Chronic expanding haematoma (CEH) is a rare type of haematoma that enlarges slowly and continuously without coagulation. In general, it gradually decreases in size and disappears naturally over time. Although the specific mechanism underlying this enlargement is not completely understood, it is thought that $\mathrm{CEH}$ is caused by minor blunt trauma or surgery [1]. In such cases, a small amount of shear stress-induced bleeding may occur in the scar tissue between the subcutaneous fat and fascia $[2,3]$. The bleeding then continues, and the haematoma enlarg- es because of the accumulation of coagulation factors and degradation products of the fibrinolytic system [1]. Furthermore, because of irritation, blood and breakdown products could cause repeated exudation or bleeding from capillaries in the granulation tissue [4].

CEH can occur at any location in the body [5], including the calf, thigh [6], knee [7], buttocks [8], abdomen, pelvis [9], and breast [10], and may often develop in simulated neoplasms. It occurs not only in soft tissues but also in any type of cavity. It is presumed that shear force is easily generated in the back region during daily activities such as turning over during sleep and 
twisting at the waist while walking [5]. Therefore, reconstructive surgeons should be aware that $\mathrm{CEH}$ can develop in patients who undergo surgery with a latissimus dorsi flap. Three such cases of $\mathrm{CEH}$ at the latissimus dorsi flap site have been previously reported $[5,11,12]$. Although treatment for $\mathrm{CEH}$ has not yet been established, surgical excision or aspiration is commonly performed. Here, we report three cases of CEH that developed at the latissimus dorsi flap donor site between August 2012 and August 2014 at our institute; these cases improved following a triamcinolone injection, without any severe complications.

\section{CASES}

Three female patients were included in this study. Data on exposures and outcomes were obtained using chart review after the patient provided informed consent. Although the patients did not have any obvious complications immediately after the flap surgery, $\mathrm{CEH}$ developed in the donor site at a mean of 47 months (range, 9 months -6 years) postoperatively. This study was performed according to institutional ethical guidelines.

\section{Treatment procedure}

In the first two patients, the initial treatment consisted of multiple sessions of aspiration of the $\mathrm{CEH}$ in the outpatient clinic. The haematoma was drained using an indwelling needle and syringe, without the use of any anaesthesia, while the patients were seated. Then, with the patients lying in the prone position, a piece of cotton ( $5 \mathrm{~cm}$ thickness) was applied directly onto the site of $\mathrm{CEH}$, a piece of gauze was laid on top of the cotton, and an elastic bandage (width, $10 \mathrm{~cm}$ ) was wrapped over the cotton and gauze and around the body to compress the site to prevent recurrence. The patients were carefully instructed on how to change the cotton and elastic bandage themselves at home after washing. The compression dressing was basically applied 24 hours a day. However, the $\mathrm{CEH}$ continued to enlarge. Therefore, after draining the haematoma, $40 \mathrm{mg}$ of triamcinolone diluted in $4 \mathrm{~mL}$ of saline was immediately injected into the draining space by using another syringe through the same indwelling needle. The same compression dressing was tightly applied for at least 1 month to ensure appropriate spread of triamcinolone and prevent recurrence. In the third patient, a 40-mg triamcinolone injection was administered immediately after the initial drainage of the haematoma, without first attempting multiple sessions of aspiration.

\section{Case 1 (42-year-old woman)}

The patient (height, $163 \mathrm{~cm}$; weight, $54.0 \mathrm{~kg}$ ) had undergone left mastectomy for breast cancer (T1NOM0 stage 1) at 39 years of age, and reconstruction surgery using a latissimus dorsi flap was performed after 4 months. The patient also suffered from systemic lupus erythematosus and was taking aspirin for thromboprophylaxis. Although the postoperative course was uneventful and no history of trauma was evident, a large mass on the back measuring $100 \mathrm{~mm} \times 30 \mathrm{~mm} \times 230 \mathrm{~mm}$ suddenly developed 9 months after the breast reconstruction surgery (Fig. 1A). The location corresponded with the previous latissimus dorsi flap donor site. The mass was diagnosed with $\mathrm{CEH}$, and the haematoma was aspirated using an indwelling needle; the total aspiration volume was $130 \mathrm{~mL}$. The $\mathrm{CEH}$ contents contained

\section{Fig. 1. Case 1}

(A) A 42-year-old woman suddenly developed a large mass on the back measuring $100 \mathrm{~mm} \times 30 \mathrm{~mm} \times 230 \mathrm{~mm}, 9$ months after breast reconstruction surgery using a latissimus dorsi flap. (B) The chronic expanding haematoma contents contained blood. (C) The chronic expanding haematoma had disappeared. The image was taken 25 months after the triamcinolone injection.
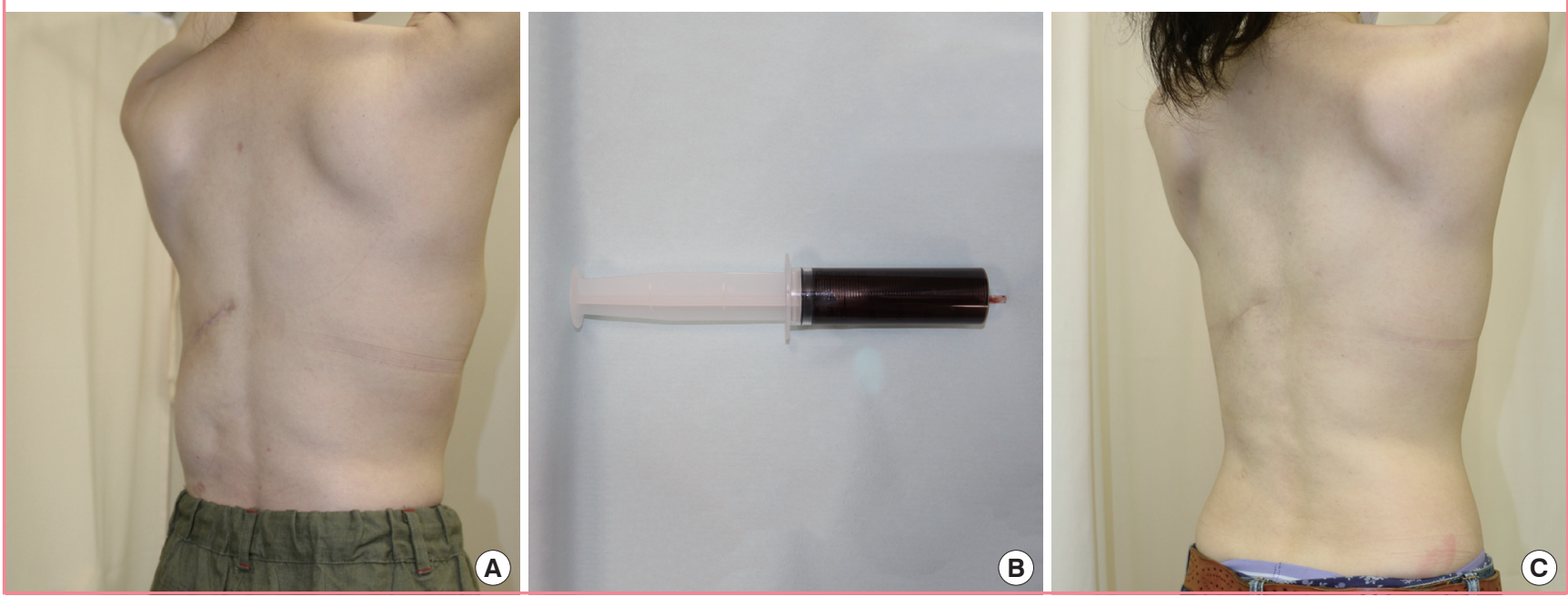


\section{Fig. 2. Case 2}

(A) Magnetic resonance imaging showed a large mass measuring $90 \mathrm{~mm} \times 75 \mathrm{~mm} \times 50 \mathrm{~mm}$ on the flap donor site. (B) The excised tissue consisted of a soft cystic mass with a thick fibrous capsule containing a blood clot and serous fluid. (C) The chronic expanding haematoma had disappeared. The image was taken 12 months after the surgery.
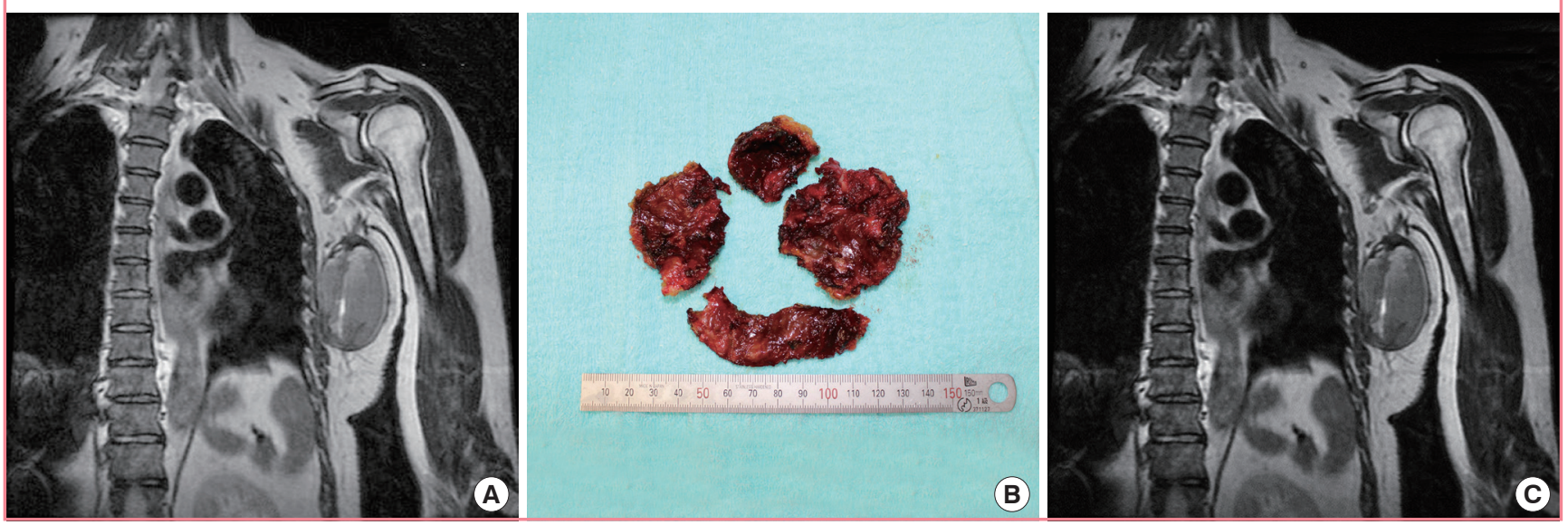

blood (Fig. 1B). Laboratory examination of the aspirated blood showed a haematocrit value of $30.6 \%$ (red blood cell [RBC] count, $320 \times 10^{4} / \mu \mathrm{L}$ ), while the haematocrit value in the blood on the same day was $34.1 \%$ (RBC count, $390 \times 10^{4} / \mu \mathrm{L}$ ). Although the patient stopped taking aspirin and the haematoma was aspirated three more times in 2 months, with aspirated volumes of $50 \mathrm{~mL}, 70 \mathrm{~mL}$, and $200 \mathrm{~mL}$, respectively, the haematoma gradually increased in size. Because $520 \mathrm{~mL}$ was aspirated at the fifth aspiration session, $40 \mathrm{mg}$ of triamcinolone was injected into the draining space followed by appropriate compression dressing for 8 weeks. The patient's condition markedly improved without further enlargement of the $\mathrm{CEH}$. Although the patient resumed taking aspirin 6 months after the triamcinolone injection, there was no recurrence or need for aspiration for 25 months (Fig. 1C).

\section{Case 2 (58-year-old woman)}

The patient (height, $158.6 \mathrm{~cm}$; weight, $70.3 \mathrm{~kg}$ ) had undergone left mastectomy for breast cancer (TisNOM0 stage 0) at 43 years of age and breast reconstruction using a latissimus dorsi flap at 52 years of age with a good postoperative course. The patient was taking sarpogrelate hydrochloride for lumbar canal stenosis. Six years after the breast reconstruction, the patient noticed a large mass measuring $90 \mathrm{~mm} \times 75 \mathrm{~mm} \times 50 \mathrm{~mm}$ on her back (Fig. 2A). The location corresponded with the previous latissimus dorsi donor site. The mass was diagnosed as $\mathrm{CEH}$, and the patient stopped taking sarpogrelate hydrochloride. The haematoma was aspirated four times in 3 months, with aspirated volumes of $100 \mathrm{~mL}, 100 \mathrm{~mL}, 130 \mathrm{~mL}$, and $190 \mathrm{~mL}$ (mean volume, $130 \mathrm{~mL}$ ). The characteristics of the $\mathrm{CEH}$ contents suggested the presence of blood. At the fourth aspiration, $40 \mathrm{mg}$ of triam- cinolone was injected into the draining space followed by compression dressing for 6 weeks. The haematoma size was reduced markedly; the aspirated fluid volume fell to $10 \mathrm{~mL}$ at 10 days after the triamcinolone injection. However, a solid haematoma, which could not be aspirated or injected with triamcinolone, remained. Hence, surgical excision of the solid haematoma was performed 2 months after the triamcinolone injection. The excised tissue consisted of a soft cystic mass with a thick fibrous capsule containing an old blood clot and serous fluid (Fig. 2B). Although the patient resumed taking sarpogrelate hydrochloride 6 months after the operation, there was no further $\mathrm{CEH}$ formation for 6 months (Fig. 2C).

\section{Case 3 (53-year-old woman)}

The patient (height, $158.0 \mathrm{~cm}$; weight, $44 \mathrm{~kg}$ ) had undergone right mastectomy for bilateral breast cancer (T1NOM0 stage 1) at the age of 48 years. Breast reconstruction using bilateral latissimus dorsi flaps was performed at the age of 49 years. Although the postoperative course was uneventful and there was no evident history of trauma, a large mass on the right back measuring $110 \mathrm{~mm} \times 60 \mathrm{~mm} \times 85 \mathrm{~mm}$ suddenly developed 5 years after the breast reconstruction surgery (Fig. $3 \mathrm{~A}$ ). The mass was diagnosed as $\mathrm{CEH}$. The location corresponded with the right latissimus dorsi donor site, and there was no sign of $\mathrm{CEH}$ on the left side. The first aspiration resulted in an aspirated volume of 60 $\mathrm{mL}$. Triamcinolone $(40 \mathrm{mg}$ ) was injected immediately after this first aspiration. Although the condition subsided for 4 months, a minor recurrence of the $\mathrm{CEH}$ was noted. Therefore, the $\mathrm{CEH}$ was again aspirated, and another 40-mg triamcinolone injection was administered. There was no further $\mathrm{CEH}$ formation for 10 months (Fig. 3B). 


\section{Fig. 3. Case 3}

(A) A 53-year-old woman developed a large mass on the right back measuring $110 \mathrm{~mm} \times$ $60 \mathrm{~mm} \times 85 \mathrm{~mm}$ (surrounded by yellow arrows) 5 years after bilateral breast reconstruction surgery using bilateral latissimus dorsi flaps. (B) The chronic expanding haematoma had disappeared. The image was taken 10 months after the triamcinolone injection.
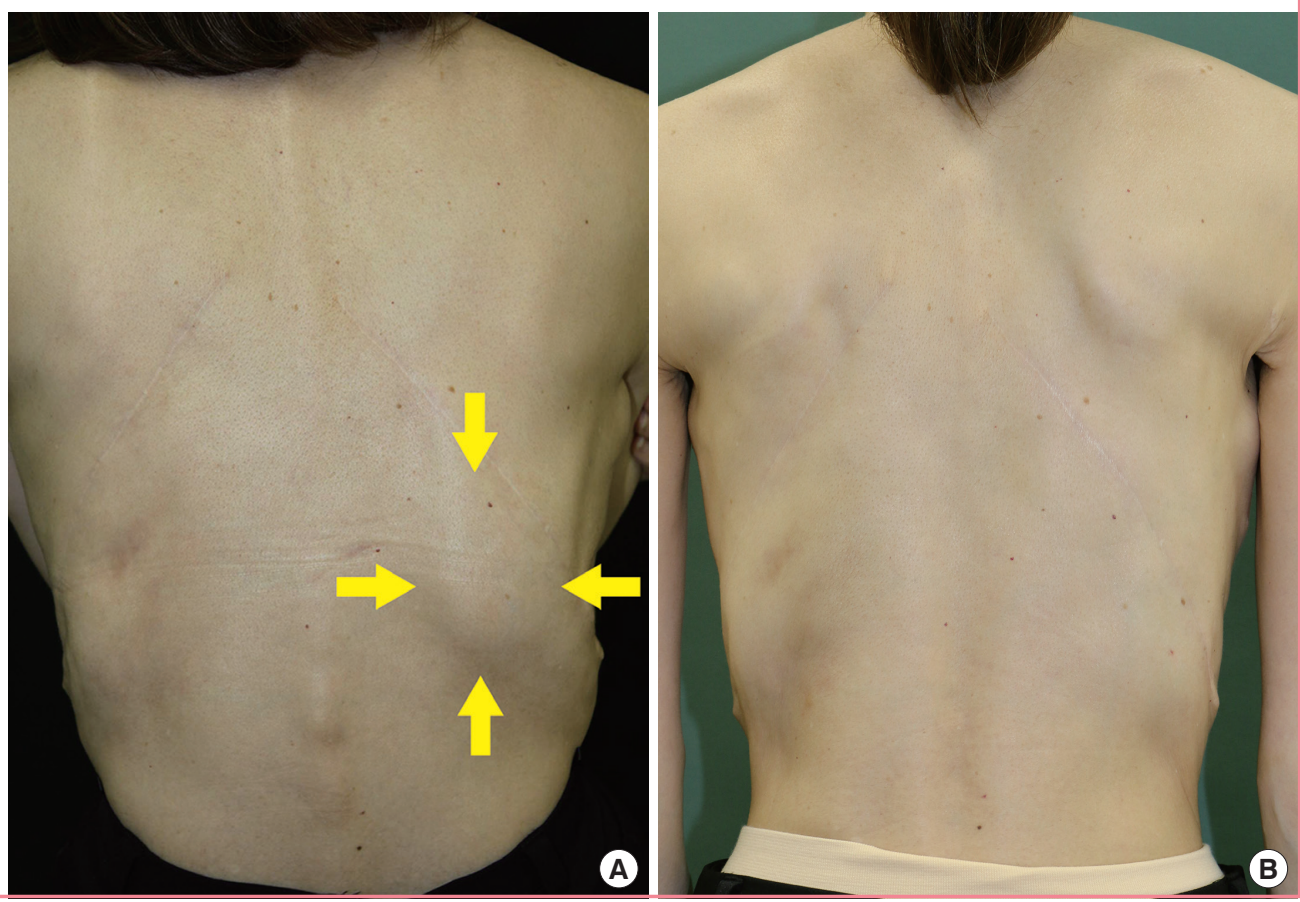

\section{DISCUSSION}

The latissimus dorsi is a major muscle of the back. A large space is created when a portion of the latissimus dorsi muscle is harvested. In this space, a small amount of bleeding might occur due to shear stress in the scar tissue between the subcutaneous fat and fascia. Three cases of $\mathrm{CEH}$ in the latissimus dorsi flap donor site, with no noted history of trauma, have been previously reported $[5,11,12]$. CEH developed 1 month, 5 years, and 13 years after the flap surgery in these three cases. In our series, the haematoma developed 9 months, 5 years, and 6 years after the flap surgery in the three cases. Based on the findings of these cases, surgeons should consider that the duration between flap surgery and the development of $\mathrm{CEH}$ may range from months to years.

In our first two cases, antiplatelet medication was administered before the operation. Acetylsalicylic acid (Bayaspirin) was orally administered in case 1; this medication is used primarily in the treatment of cardiovascular diseases, such as myocardial infarction and cerebral infarction. It acts by inhibiting cyclooxygenase and plays an important role in platelet aggregation; it acts as an antiplatelet agent by inhibiting thromboxane A2 [13]. Sarpogrelate hydrochloride (Anplag) was orally administered in case 2. It inhibits platelet aggregation and vessel constriction by selectively antagonising the serotonin receptors 5-HT2 on the vessels and platelets [14]. This improves the ischemic symptoms of chronic arterial occlusive disease, such as ulcers, pain, and cold sensation. The main side effect of these antiplatelet agents is a bleeding tendency, which could increase the risk of a postoperative haematoma, including $\mathrm{CEH}$.

There are few reports regarding the treatment for $\mathrm{CEH}$. However, because $\mathrm{CEH}$ is usually encapsulated, surgical excision or aspiration is commonly used. As described here, a triamcinolone injection could be the treatment of choice, particularly after failed aspiration attempts. Triamcinolone has previously been used to improve seroma at the latissimus dorsi flap donor site [15]. Despite a major difference in blood cell concentration, the clinical conditions of $\mathrm{CEH}$ and seroma after latissimus dorsi flap harvesting are relatively similar; both involve the accumulation of liquid between the skin and the underlying scar tissue and occur because of inflammation caused by postoperative shear stress. In seroma, inflammatory mediators are stimulated by tissue damage, and the exudate stagnates in the dead space. Triamcinolone is an anti-inflammatory agent that is believed to reduce the re-accumulation of a seroma. It suppresses the inflammatory process by the formation of the phospholipase inhibitor lipocortin, which diminishes the supply of arachidonic acid available for synthesis of prostaglandin and leukotrienes. As a result, capillary permeability, oedema, migration of leucocytes, and the subsequent signs of capillary proliferation and fibroblast and collagen deposition are inhibited. In $\mathrm{CEH}$, the pathogenesis is considered to involve inflammation that is induced by the destructive products of hemocytes [1]. Therefore, we concluded that a localised triamcinolone injection might also be effective for $\mathrm{CEH}$. 
Previous reports indicate that $40-80 \mathrm{mg}$ of triamcinolone should be injected in cases of seroma. On the basis of these reports, we injected $40 \mathrm{mg}$ of triamcinolone at each session. Consequently, the haematoma volumes were reduced after the initial injection. Case 1 showed a marked improvement without the need for an additional triamcinolone injection, even though this case involved the largest haematoma $(520 \mathrm{~mL})$ among the three cases. The fluid volume of $\mathrm{CEH}$ also decreased in case 2 , although a solid haematoma remained. Surgical resection was required for the solid haematoma. The recurrence experienced by case 3 after the first triamcinolone injection resolved with a second $40 \mathrm{mg}$ triamcinolone injection. On the basis of these experiences, we recommend an initial 40-mg triamcinolone injection at the first session irrespective of the haematoma size. For recurrence, a second 40-mg triamcinolone injection is suggested. However, the most appropriate triamcinolone dose for $\mathrm{CEH}$ treatment should be assessed further with a larger number of cases.

In conclusion, in cases of $\mathrm{CEH}$, a triamcinolone injection, rather than surgical excision or aspiration, can be considered for treatment.

\section{REFERENCES}

1. Yamamoto S, Momose T, Aoyagi M, et al. Spontaneous intracerebral hematomas expanding during the early stages of hemorrhage without rebleeding. Report of three cases. J Neurosurg 2002;97:455-60.

2. Bradshaw JR, Davies GT, Edwards PW, et al. The radiological demonstration of traumatic cysts due to severe soft tissue trauma. Br J Radiol 1972;45:905-10.

3. Sterling A, Butterfield WC, Bonner R Jr, et al. Post-traumatic cysts of soft tissue. J Trauma 1977;17:392-6.

4. Labadie EL, Glover D. Physiopathogenesis of subdural hematomas. Part 1: Histological and biochemical comparisons of subcutaneous hematoma in rats with subdural hematoma in man. J Neurosurg 1976;45:382-92.

5. Watanabe K, Morihisa Y, Gotanda K, et al. A case of a chron- ic expanding hematoma that developed in a latissimus dorsi muscle flap donor site. J Plast Reconstr Aesthet Surg 2013; 66:872-4.

6. Goddard MS, Vakil JJ, McCarthy EF, et al. Chronic expanding hematoma of the lateral thigh and massive bony destruction after a failed total hip arthroplasty. J Arthroplasty 2011; 26:338.e13-5.

7. Sreenivas M, Nihal A, Ettles DF. Chronic haematoma or soft-tissue neoplasm? A diagnostic dilemma. Arch Orthop Trauma Surg 2004;124:495-7.

8. Kitagawa Y, Yokoyama M, Tamai K, et al. Chronic expanding hematoma extending over multiple gluteal muscles associated with piriformis syndrome. J Nippon Med Sch 2012; 79:478-83.

9. Sakurai J, Akaki S, Yonezawa M, et al. Intrapelvic chronic expanding hematoma: magnetic resonance imaging findings with pathological correlation. Magn Reson Med Sci 2010; 9:81-4.

10. Daw JL, Lewis VL, Smith JW. Chronic expanding hematoma within a periprosthetic breast capsule. Plast Reconstr Surg 1996;97:1469-72.

11. Lewis VL Jr, Johnson PE. Chronic expanding hematoma. Plast Reconstr Surg 1987;79:465-7.

12. Sugaya F, Kitazawa T, Fukuda C, et al. Chronic expanding haematoma of the latissimus dorsi muscle flap donor site: a case report. Jpn J Plast Surg 2008;51:1205-11.

13. Pulcinelli FM, Riondino S, Celestini A, et al. Persistent production of platelet thromboxane $\mathrm{A} 2$ in patients chronically treated with aspirin. J Thromb Haemost 2005;3:2784-9.

14. Satoh K, Yatomi Y, Ozaki Y. A new method for assessment of an anti-5HT(2A) agent, sarpogrelate hydrochloride, on platelet aggregation. J Thromb Haemost 2006;4:479-81.

15. Taghizadeh R, Shoaib T, Hart AM, et al. Triamcinolone reduces seroma re-accumulation in the extended latissimus dorsi donor site. J Plast Reconstr Aesthet Surg 2008;61:63642. 